\title{
The Maximum Drawdown of the Brownian Motion
}

\author{
Malik Magdon-Ismail \\ Dept Computer Science \\ Rensselaer Polytechnic Institute \\ Room 207, Lally Bldg \\ 1108 th Street \\ Troy, NY 12180 \\ magdon@cs.rpi.edu \\ Amir Atiya \\ Dept Computer Engineering \\ Cairo University \\ Giza, Egypt \\ amiratiya@link.net \\ Amrit Pratap \\ Dept Computer Science \\ Caltech 136-93 \\ Pasadena, CA 91125 \\ Yaser Abu-Mostafa \\ Dept Electrical Engineering \\ Caltech 136-93 \\ Pasadena, CA 91125
}

The MDD is defined as the maximum loss incurred from peak to bottom during a specified period of time. Mathematically it is given by

$$
M D D(T)=\sup _{t \in[0, T]}\left[\sup _{s \in[0, t]} X(s)-X(t)\right]
$$

where $X(t)$ represents the equity curve of the trading system or fund. The maximum drawdown MDD is the most widespread risk measure among money managers and hedge funds. It is often preferred over some of the other risk measures because of the tight relationship between large drawdowns and fund redemptions. Also, a large drawdown can even indicate the start of a deterioration of an otherwise successful trading system, for example due to a market regime switch.

Despite the importance of such a problem, there has been a lack of theoretical results covering that problem. In this article, we develop new analytic formulas that relate a 
trading strategy's return, standard deviation of returns, and MDD. A widely followed measure of risk adjusted return based on MDD is the Sterling Ratio. It is defined as

$$
\text { Sterling }(T)=\frac{\text { Return over }[0, T]}{M D D \text { over }[0, T]}
$$

It basically measures how much return to expect for every unit of downside risk, akin to the widely used Sharpe ratio. In an environment where markets exhibit frequent crashes and portfolios encounter sustained periods of losses the Sterling ratio has emerged as one of the leading performance measures used in the industry. In this article we also develop formulas that relate the Sterling ratio with the Sharpe ratio.

The only currently available way to analyze MDD is to measure it on a previous historical period. The problem with this approach is that this represents only a onesample-point estimate. Our developed formulas will give the expected MDD in a specific time period for a given return and standard deviation. As such, they will provide some guidance as to what MDD to expect and also whether a trading system with a given observed MDD is considered a "favorable" or "unfavorable" system.

Assume a trading system follows a Brownian motion:

$$
d x=\mu d t+\sigma d w \quad 0 \leq t \leq T
$$

where $\mu$ is the average return per unit time, $\sigma$ is the standard deviation of the returns per unit time for the trading system, and $d w$ is the usual Wiener increment. This model assumes that profits are not reinvested using the trading model. If profits are reinvested, then the Geometric Brownian motion is the appropriate model. For such a case equivalent formulas can similarly be derived by taking a log transformation. (The MDD in that case is defined as the percentage loss rather than absolute loss.) There could potentially be trading systems that have different or more complicated process models. However, the benefit from considering a standard Brownian motion is that the resulting formulas could provide a baseline benchmark, against which we can compare any given system.

A starting step in our derivations has been to show that the drawdown process is equivalent to a Brownian motion with drift $-\mu$ and one absorbing barrier and one reflecting barrier. That called for the first passage time distribution for such a process. We utilize the formulas for that first passage distribution, given by [1] and [2]. We analyzed both the continuous time case and the discrete case, and established that the continuous case can be obtained as the limiting value of the discrete time case. According to our derivations, as it turned out, the cases of $\mu>0, \mu=0$, and $\mu<0$ are qualitatively different and result in formulas that are quite different. The formulas for the expected MDD are as follows: 


$$
E(M D D)= \begin{cases}\frac{\sigma^{2}}{\mu} Q_{p}\left(\frac{\mu^{2} T}{\sigma^{2}}\right) & \text { if } \mu>0 \\ 1.2552 \sigma \sqrt{T} & \text { if } \mu=0 \\ \frac{\sigma^{2}}{\mu} Q_{n}\left(\frac{\mu^{2} T}{\sigma^{2}}\right) & \text { if } \mu<0\end{cases}
$$

The functions $Q_{n}$ and $Q_{p}$ are integrals of series expansions that can be evaluated numerically. They are given by the following integrals:

$$
\begin{aligned}
Q_{p}(x)= & 2 \int_{0}^{\infty}\left[e^{-u} \sum_{n=1}^{\infty} \frac{\sin ^{3}\left(\theta_{n}\right)}{\theta_{n}-\cos \left(\theta_{n}\right) \sin \left(\theta_{n}\right)}\left(1-e^{-\frac{x}{\cos ^{2}\left(\theta_{n}\right)}}\right)\right. \\
& \left.+e^{-\frac{u}{\tanh (u)}} \sinh (u)\left(1-e^{-\frac{x}{2 \cosh ^{2}(u)}}\right)\right] d u \\
Q_{n}(x)= & -2 \int_{0}^{\infty} e^{u} \sum_{n=1}^{\infty} \frac{\sin ^{3}\left(\theta_{n}\right)}{\theta_{n}-\cos \left(\theta_{n}\right) \sin \left(\theta_{n}\right)}\left(1-e^{-\frac{x}{2 \cos ^{2}\left(\theta_{n}\right)}}\right) d u
\end{aligned}
$$

where $\theta_{n}$ is the solution of the eigenvalue problem

$$
\tan \left(\theta_{n}\right)=\frac{\theta_{n}}{u} \quad \theta_{n} \in\left(\left(n-\frac{1}{2}\right) \pi,\left(n+\frac{1}{2}\right) \pi\right)
$$

We note that care has to be excercized when numerically evaluating them (especially $Q_{n}$ ) because the series converge rather slowly. However, the interesting thing here is that $Q_{n}$ and $Q_{p}$ do not depend on any constant or quantity such as $\mu, \sigma$, or $T$. As such, they are universal functions that can be evaluated once and for all. Then, they can be tabulated and used in a lookup manner (rather than evaluated numerically every time they are used). Figures 1 and 2 show plots for $Q_{p}$ and $Q_{n}$ respectively.

From the formulas, one can deduce a relationship between the MDD and the wellknown Sharpe ratio. The Sharpe ratio is given by $\mu / \sigma$ where $\mu$ and $\sigma$ are measured in units of one year. One can deduce that for the case $\mu>0$ :

$$
E(M D D)=\frac{\sigma}{\text { Sharpe }} Q_{p}\left((\text { Sharpe })^{2} T\right)
$$

Now comes the question, how does the drawdown behave asymptotically? Most trading desks are interested in long term performance results. That is, what system can survive over the long period ahead, and provide superior return with a level of drawdown that is acceptable and does not threaten its survival. We have derived formulas for the drawdown for $T \longrightarrow \infty$ ( $T$ is the investment horizon). They are given by the following theorem: 


\section{Theorem:}

The limiting expectation as $T \longrightarrow \infty$ is given by.

$$
\left.E(M D D)\right|_{T \rightarrow \infty}= \begin{cases}\frac{\sigma^{2}}{\mu}\left(--0.5416+0.5 \log (T)+\log \frac{\mu}{\sigma}\right) & \text { if } \mu>0 \\ 1.2552 \sigma \sqrt{T} & \text { if } \mu=0 \\ -\mu T & \text { if } \mu<0\end{cases}
$$

One can see that the drawdown can scale in three different ways $(\log (T), \sqrt{T}$, or $T)$ depending on the regime of $\mu$. It is as if the problem undergoes a "phase shift" as $\mu$ goes from negative to positive. It cain therefore be used as a sort of hypothesis test: is the return positive, even or negative? This observation also supports the importance of the drawdown as an indicator of the effectiveness of a trading strategy.

We introduced earlier in the article the Sterling Ratio. Mathematically it is defined as

$$
\text { Sterling }(T)=\frac{\mu}{E(M D D(T))}
$$

where $M D D(T)$ represents the maximum drawdown in an interval of length $T$. Focusing our attention to the more interesting case of $\mu>0$, we get

$$
\operatorname{Sterling}(T)=\frac{(\text { Sharpe })^{2}}{Q_{p}\left((\text { Sharpe })^{2} T\right)}
$$

Considering also the asymptotic case $T \longrightarrow \infty$,

$$
\text { Sterling }(T \longrightarrow \infty)=\frac{(\text { Sharpe })^{2}}{-0.5416+0.5 \log (T)+\log (\text { Sharpe })}
$$

These formulas indicate a direct relationship between the Sterling Ratio and the Sharpe Ratio. A deviation observed from historical data indicates a tendency to either have or avoid excessive successive loss periods. This feature depends on the trading system, the types of markets (for example the trendiness or mean reversion tendency of the markets), and the degree of diversification.

Overall, the MDD is a very important risk measure. To be able to use it more insightfully, its analytical properties have to be understood. As a step towards this direction, we have presented in this article some analytic results that we have developed. We hope more and more results will come out from the research community analyzing this important measure. 


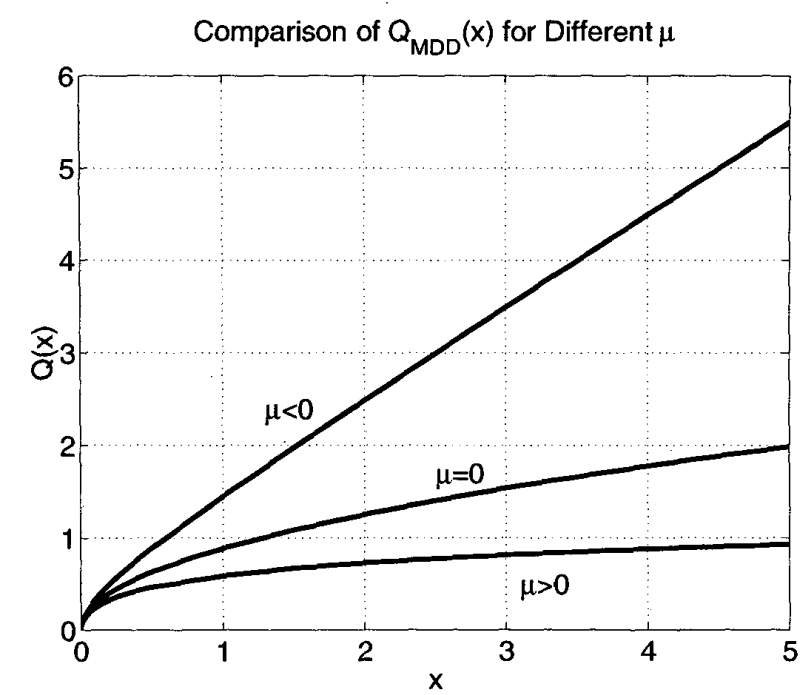

Figure 1: Behavior of the $Q$ functions for different $\mu$.

\section{References:}

[1] M. Domine, "First passage time distribution of a Wiener process with drift concerning two elastic barriers", Journal of Applied Probability, 33:164-175, 1996.

[2] Blasi, "On a random walk between a reflecting and an absorbing barrier", annals of Probability, 4(4):695-696, 1976. 